九州大学学術情報リポジトリ

Kyushu University Institutional Repository

\title{
STABLE CONFIGURATIONS IN CELL SPACE UNDER THE THRESHOLD TYPE TRANSITION RULE
}

Sasagawa, Fumiyoshi

International Institute for Advanced Study of Social Information Science, Fujitsu Ltd.

https://doi.org/10.5109/13409

出版情報: Bulletin of informatics and cybernetics. 24 (1/2), pp.39-52, 1990-03. Research Association of Statistical Sciences

バージョン :

権利関係 : 


\title{
STABLE CONFIGURATIONS IN CELL SPACE UNDER THE THRESHOLD TYPE TRANSITION RULE
}

By

Fumiyoshi SaSagawa*

\begin{abstract}
This paper is one of a series of the papers treating stable configurations in cell space. The strong-week relations between states are introduced in the local transition rule of the cell space. The local transition rule is systematically represented by the threshold type function. The pattern of stable configuration is remarkably changed when the thresholds of the local transition rule exceeds some critical values. It is shown that the stable configurations in the $m \times n$ cell space are dependent on both the size of the basic cell space and the type of local transition rule. Hierarchy of types of local transition rules and cluster property of stable configurations are obtained. In addition the singular stable subconfiguration is defined and it is found that a stable configuration becomes another stable configuration if a state of the singular stable subconfiguration is changed.
\end{abstract}

\section{Introduction}

Life has various characteristics and has been analyzed mathematically, for example, von Neumann [1] has proposed self-reproduced automata. Recently, pattern formations of cellular automata has been investigated by many authors [2]. Such approaches may be included in biomathematics. Kitagawa proposed that biomathematics consists of five elements: discrete mathematics, combinatorial mathematics, dynamical mathematics, evolutionary mathematics and design mathematics [3], which are necessary to understand biological phenomena. In the beginning, he started the study of cell space. T. Kitagawa and M. Yamaguchi $[4,5]$ studied the $m \times n$ cell space. In their $m \times n$ cell space, the transition of a configuration is caused by a change of configuration of a $2 \times 2$ cell subspace named basic cell space, which is subject to the local majority transformation. By them, it was obtained that any initial configuration becomes some stable configuration which is a checked pattern after the long time [5]. With respect to transition of configuration, important mathematical notions, for example, stable configuration, garden of Eden configuration, determinative cell subspace and elementary stable configuration were introduced in cell space by them [5-8]. Recently, we extended their results to a cell space with three or four states and investigated oscillation phenomena of the configuration in a cell space under a fixed boundary condition [9].

\footnotetext{
* International Institute for Advanced Study of Social Information Science, Fujitsu Ltd. 17-25, Shinkamata 1-chome, Ohta-ku, Tokyo 144, Japan
} 
In this paper, we investigate the interrelation between the size of basic cell space and stable configuration. We propose a threshold type transition rule, which is defined by the threshold function and the weights of states. In general, our local transition rule is not symmetric in respect to states. From this property, this rule represents a strong-weak relation between states. The cell space with this rule is regarded as a mathematical model of ecosystem with strong and weak species. Types of local transition rules are derived from this local transition rule.

\section{The Local Transition Rule and Strong-Weak Relation of States}

The interrelations between the size of basic cell space and stable configuration are investigated in this paper. For this purpose, it is enough that each cell $c_{i, j}(1 \leqslant i \leqslant m$; $1 \leqslant j \leqslant n)$ takes one of two states $\{a, b\}$. A basic cell space is a cell subspace in which a local transition rule described below is applied $[4,5]$. In our $m \times n$ cell space, a basic cell space is a $k \times l$ cell subspace $(2 \leqslant k \leqslant m ; 2 \leqslant l \leqslant n)$. The set of all basic cell spaces is denoted by $\mathscr{B}$. Hereafter, we use a notation $T[X]$ of a restricted configuration over the cell subspace $T$ where $X$ is a whole configuration of the $m \times n$ cell space. In order to define the local transition rule, we introduce the following counting function.

$$
f\left(N_{a}, N_{b}\right)=\frac{w_{a} N_{a}-w_{b} N_{b}}{w_{a}+w_{b}}
$$

where $w_{a}>0, w_{b}>0$. Here, we introduce weights $w_{a}$ and $w_{b}$ corresponding to states " $a$ " and " $b$ " and $N_{a}\left(N_{b}\right)$ is the number of state " $a$ " (" $b$ ") in a basic cell space $B \in \mathscr{B}$. Then, the local transition rule $L T$ is defined by the following manner:

Transition $L T_{a}$. If $f\left(N_{a}, N_{b}\right)>\eta_{a}$,

$$
B[X] \rightarrow\left[\begin{array}{cccc}
a & a & \cdots & a \\
a & a & \cdots & a \\
\vdots & \vdots & \ddots & \vdots \\
a & a & \cdots & a
\end{array}\right] .
$$

Transition $L T_{b}$. If $f\left(N_{a}, N_{b}\right)<\eta_{b}$,

$$
B[X] \rightarrow\left[\begin{array}{cccc}
b & b & \cdots & b \\
b & b & \cdots & b \\
\vdots & \vdots & \ddots & \vdots \\
b & b & \cdots & b
\end{array}\right]
$$

Transition $L T_{n}$. Otherwise,

$$
B[X] \rightarrow B[X]
$$

In the above local transition rule, $\eta_{a}$ and $\eta_{b}$ are thresholds and $\eta_{b} \leqslant \eta_{a}$. The values of two weights are considered as a representation of relative strongness between states. For example, if the value of $w_{a}$ is much larger than $w_{b}$, the transition $L T_{a}$ occurs and the 
basic cell space is overwhelmed by states " $a$ ". Thus, the local transition rule is not symmetric in respect to two states. The disjoint basic cell spaces $B_{1}, B_{2}, \ldots, B_{r}$ are randomly selected and the above local transition rule is applied to these basic cell spaces $[4,5]$. If definite values of weights and thresholds are given, we have a table of transitions for all possible pairs $\left(N_{a}, N_{b}\right)$. Let's call this table a type of local transition rule or simply a rule.

\section{Types of Local Transition Rules for $k \times l$ Basic Cell Space}

In the cell space with $k \times l$ basic cell spaces, the order of values of the counting function can be derived as

$$
f_{0}<f_{1}<f_{2}<\cdots<f_{k l-1}<f_{k l} .
$$

Here, we use the abbreviation that $f_{i}=f(i, k l-i)$. From the above order and the definition of local transition rule (2-2), (2-3) and (2-4), we can obtain all types of local transition rules. In order to present all types of local transition rules, we define two non-negative integers $r_{1}$ and $r_{2}$ such that $r_{1}=\max \left\{j \mid f_{j}<\eta_{b}, 0 \leqslant j \leqslant k l\right\}$ and $r_{2}=$ $\min \left\{j \mid \eta_{a}<f_{j}, 0 \leqslant j \leqslant k l\right\}$. Furthermore, if $\eta_{b}<f_{0}$, then we define that $r_{1}=\perp$ and similarly, if $f_{k l}<\eta_{a}$, then $r_{2}=1$. By these $r_{1}$ and $r_{2}$, types of local transition rules are completely determined. The corresponding rule is called $\left[r_{1}, r_{2}\right]$-rule. We present the following types of local transition rules.

1. $[\perp, 0]$-rule (All-a rule). For any pair $\left(N_{a}, N_{b}\right)$, the transition $L T_{a}$ takes place.

2. $[k l, \perp]$-rule (All-b rule). For any pair $\left(N_{a}, N_{b}\right)$, the transition $L T_{b}$ takes place.

3. $[q-1, q]$-rule $(1 \leqslant q \leqslant k l-1)$. If $N_{a} \geqslant q$, then the transition $L T_{a}$ takes place. If $N_{a}<q$, then the transition $L T_{b}$ takes place.

4. $[p-1, p+d]$-rule $(1 \leqslant p \leqslant k l-1,1 \leqslant d \leqslant k l-p)$. Two natural numbers are defined by the following way.

$$
N_{1}=p, \quad N_{2}=d+N_{1}-1 .
$$

If $N_{a}>N_{2}$, then the transition $L T_{a}$ takes place. If $N_{a}<N_{1}$, then the transition $L T_{b}$ takes place. If $N_{1} \leqslant N_{a} \leqslant N_{2}$, then the transition $L T_{n}$ take place.

Here, $d$ of $[p-1, p+d]$-rule denotes the number of transition $L T_{n}$ in this rule. It should be noted that the number of transition $L T_{n}$ in $[q-1, q]$-rule is zero.

\section{Stable Configurations for $k \times l$ Basic Cell Spaces under $[\perp, 0],[k l, \perp],[q-1, q]$, and $[p-1, p+1]$-rule}

In an $m \times n$ cell space with $k \times l$ basic cell spaces, the stable configuration $X_{S}$ is defined by the same way as T. Kitagawa and M. Yamaguchi. If and only if $\operatorname{LT}\left(B\left[X_{S}\right]\right)$ $=B\left[X_{S}\right]$ for any $B \in \mathscr{B}, X_{S}$ is said to be stable $[4,5]$. Under $[\perp, 0]([k l, \perp])$-rule, we find that $X_{A}\left(X_{B}\right)$ configuration is only one stable configuration where a configuration is said to be an $X_{A}\left(X_{B}\right)$ configuration if and only if all states are " $a$ "(" $b$ "). Furthermore, under $[q-1, q]$-rule, all stable configurations are $X_{A}$ and $X_{B}$ configurations. These rules don't contain a transition $L T_{n}$ and any $B[X]$ for $B \in \mathscr{B}$ changes into a uniform 
configuration of $B$ by an application of any of these rules. Thus, stable configurations are simple $X_{A}$ and $X_{B}$.

The set of all stable configurations $\mathscr{X}_{S}$ consists of isolated and non-isolated stable configurations [8]. Here, the isolated stable configuration is a stable configuration without direct ancestor [8]. The non-isolated stable configuration has a direct ancestor [8]. Next, we consider non-isolated stable configurations under $[p-1, p+1]$-rule. Then, we get regular patterns of non-isolated stable configurations. These patterns depends on the size of basic cell space and rule. For giving a description of these stable configurations, we define a set of cell subspaces $\mathscr{M}_{p}^{(u)}$ and $\mathcal{N}_{p}^{(v)}(u, v \in \mathbf{N})$. We define a cell subspace $R_{\alpha, j}^{p}$ as

$$
R_{\alpha, j}^{p}=\left\{c_{\alpha_{1}, j}, c_{\alpha_{2}, j}, \ldots, c_{\alpha_{p}, j}, c_{\alpha_{1}+k, j}, c_{\alpha_{2}+k, j}, \ldots, c_{\alpha_{p}+k, j}, c_{\alpha_{1}+2 k, j}, \ldots\right\},
$$

where $\alpha=\left\{\alpha_{i} ; i=1,2, \ldots, p\right\}$, and $1 \leqslant \alpha_{1}<\alpha_{2}<\cdots<\alpha_{p} \leqslant k . \quad \mathscr{M}_{p}^{(u)}$ consists of the above cell subspaces $R_{\alpha, j}^{p}$ under the condition that if and only if $R_{\alpha, j_{1}}^{p}, R_{\beta, j_{2}}^{p} \in \mathscr{M}_{p}^{(u)}$, then $\left|j_{1}-j_{2}\right| \geqslant l$. Here, the index $u$ of $\mathscr{M}_{p}^{(u)}$ corresponds to a different combination of $R_{\alpha, j}^{p}$. Similarly, a cell subpsace $C_{i, \beta}^{p}$ is defined by

$$
C_{i, \beta}^{p}=\left\{c_{i, \beta_{1}}, c_{i, \beta_{2}}, \ldots, c_{i, \beta_{p}}, c_{i, \beta_{1}+k}, c_{i, \beta_{2}+k}, \ldots, c_{i, \beta_{p}+k}, c_{i, \beta_{1}+2 k}, \ldots\right\}
$$

where $\beta=\left\{\beta_{i} ; i=1,2, \ldots, p\right\}$, and $1 \leqslant \beta_{1}<\beta_{2}<\cdots<\beta_{p} \leqslant l$. Then, all elements of $\mathcal{N}_{p}^{(v)}$ are the above cell subspace $C_{i, \beta}^{p}$ and all of these elements satisfy the condition that if and only if $C_{i_{1}, \beta}^{p}, C_{i_{2}, \beta}^{p} \in \mathscr{N}_{p}^{(v)}$, then $\left|i_{1}-i_{2}\right| \geqslant k$. Of course, the index $v$ of these sets shows that $\mathscr{N}_{p}^{(v)}$ and $\mathscr{N}_{p}^{\left(v^{\prime}\right)}$ are different sets if $v \neq v^{\prime}$.

Furthermore, as a matter of convenience, for $\mathscr{M}_{p}^{(u)}$ and $\mathscr{N}_{p}^{(v)}$, we define three kinds of cell subspaces $T_{u}^{\mathscr{M}}, T_{v}^{\mathcal{N}}$ and $T_{u, v}^{\mathscr{M}, \mathcal{N}}$.

$$
\begin{gathered}
T_{u}^{\mathscr{M}}=\bigcup_{R_{\alpha, j}^{p} \in \mathscr{M}_{p}^{(u)}} R_{x, j}^{p} \\
T_{v}^{\mathcal{N}}=\bigcup_{C_{i, \beta}^{p} \in \mathcal{N}_{p}^{(\nu)}} C_{i, \beta}^{p} \\
T_{u, v}^{\mathscr{M}, \mathcal{N}}=T_{u}^{\mathscr{M}} \cup T_{v}^{\mathcal{N}^{\mathcal{N}}}-T_{u}^{\mathscr{M}} \cap T_{v}^{\mathcal{N}}
\end{gathered}
$$

A complement of $T_{u}^{\mathscr{M}}\left(T_{v}^{\mathcal{N}}, T_{u, v}^{\mathscr{M}, \mathcal{N}}\right)$ must include at least one basic cell space and in the definition of $T_{u, v}^{\mathscr{M}, \mathcal{N}}, \mathscr{M}_{p}^{(u)}$ and $\mathscr{N}_{p}^{(v)}$ must satisfy the following conditions.

$$
R_{\alpha, j}^{p} \cap C_{i, \beta}^{p} \neq \phi
$$

for $\forall R_{\alpha, j}^{p} \in \mathscr{M}_{p}^{(u)}, \forall C_{i, \beta}^{p} \in \mathscr{N}_{p}^{(v)}$. By using the above cell subspaces, we define the following configurations.

1. The configuration $X_{S}^{\mathscr{M}, u}$ is defined by the condition:

i. If $c_{i, j} \in T_{u}^{\mathscr{M}}$, then $c_{i, j}\left[X_{S}^{\mathscr{M}, u}\right]=a$.

ii. Otherwise, $c_{i, j}\left[X_{S}^{\mathscr{M}, u}\right]=b$.

2. The configuration $X_{S}^{\mathcal{N}, v}$ is defined by the condition:

i. If $c_{i, j} \in T_{v}^{\mathcal{N}}$, then $c_{i, j}\left[X_{\mathrm{S}}^{\mathcal{N}, v}\right]=a$.

ii. Otherwise, $c_{i, j}\left[X_{S}^{\mathcal{S}, v}\right]=b$.

3. The configuration $X_{S}^{(\mathscr{H}, u),(\mathcal{N}, v)}$ is defined by the condition:

i. If $c_{i, j} \in T_{u, v}^{\mathscr{M}, \mathcal{N}}$, then $c_{i, j}\left[X_{S}^{(\mathscr{M}, u),(\mathcal{N}, v)}\right]=a$.

ii. Otherwise, $c_{i, j}\left[X_{\mathrm{S}}^{(\mathscr{M}, \mu),(\mathcal{N}, v)}\right]=b$. 


\section{1. $\max (k, l)<p<k l-\max (k, l)$}

In this case, the non-isolated stable configurations are very simple but the isolated stable configurations become more irregular. Here, we present only the pattern of the non-isolated stable configurations.

THEOREM 1. In the $m \times n$ cell space with $k \times l$ basic cell space, if $\max (k, l)<p<$ $k l-\max (k, l)$, then under $[p-1, p+1]$-rule the non-isolated stable configurations are only $X_{A}$ and $X_{B}$ configurations. ${ }^{\dagger}$

Proof. If $X_{S}$ is a non-isolated stable configuration, there exists one basic cell space $B$ such that $B\left[X_{S}\right]$ consists of only the same state. Firstly, we consider the case that all states of $B\left[X_{S}\right]$ are " $b$ ". We assume that $B$ has the following cells:

$$
B=\left[\begin{array}{cccc}
c_{i, j} & c_{i, j+1} & \cdots & c_{i, j+l-1} \\
c_{i+1, j} & c_{i+1, j+1} & \cdots & c_{i+1, j+l-1} \\
\vdots & \vdots & \ddots & \vdots \\
c_{i+k-1, j} & c_{i+k-1, j+1} & \cdots & c_{i+k-1, j+l-1}
\end{array}\right] .
$$

The set of upper cells of $B$ is

$$
S_{1}=\left\{c_{i-1, j}, c_{i-1, j+1}, \ldots, c_{i-1, j+l-1}\right\} .
$$

The sets of lower, left and right cells of $B \in \mathscr{B}$ are respectively

$$
\begin{aligned}
& S_{2}=\left\{c_{i+k, j}, c_{i+k, j+1}, \ldots, c_{i+k, j+l-1}\right\}, \\
& S_{3}=\left\{c_{i, j-1}, c_{i+1, j-1}, \ldots, c_{i+k-1, j-1}\right\}, \\
& S_{4}=\left\{c_{i, j+l}, c_{i+1, j+l}, \ldots, c_{i+k-1, j+l}\right\} .
\end{aligned}
$$

For example, let's take the basic cell space $B_{1}$ that consists of a sum of $S_{1}$ and a subset $\left\{c_{x, y} \mid i \leqslant x \leqslant i+k-2, j \leqslant y \leqslant j+l-1\right\}$ of $B$. That is, $B_{1}=\left\{c_{r, s} ; i-1 \leqslant r \leqslant i+k-2\right.$, $j \leqslant s \leqslant j+l-1\}$. Then, by the condition that $\max (k, l)<p<k l-\max (k, l)$, we can not allocate the state " $a$ " to these upper cells because we can not get a stable configuration of $B_{1}$ even if " $a$ " state is allocated to all cells of $B_{1}$. Thus, all states of $S_{1}\left[X_{S}\right]$ should be " $b$ ". For $S_{2}, S_{3}$, and $S_{4}$, the similar arguments holds. Furthermore, it is easily found that states of $c_{i-1, j-1}, c_{i-1, j+l}, c_{i+k, j-1}, c_{i+k, j+l}$ are " $b$ ". By continuing above arguments step by step, we obtain $X_{B}$ configuration. Secondly, in the case that all states of $B\left[X_{S}\right]$ are " $a$ ", by using the similar arguments, we obtain $X_{A}$ configuration.

Q.E.D.

\section{2. $\min (k, l)<p \leqslant \max (k, l)$}

In this case, there are patterns of non-isolated stable configurations other than $X_{A}$ and $X_{B}$ configurations. By using the definitions of the above sets, we can give a pattern of non-isolated stable configuration in the case that $k \geqslant 2, l \geqslant 3(k \geqslant 3, l \geqslant 2)$.

\footnotetext{
+ It should be noted that this theorem holds for $\left[r_{1}, r_{2}\right]$-rule under the condition that $\max (k, l) \leqslant r_{1}<$ $r_{2} \leqslant k l-\max (k, l)$. This is due to the referee's comment.
} 
THEOREM 2. If $k>l$, in the $m \times n$ cell space with $k \times l$ basic cell spaces $(k \geqslant 3$, $l \geqslant 2$ or $k \geqslant 2, l \geqslant 3$ ), non-isolated stable configurations are $X_{A}, X_{B}$ and $X_{S}^{\mathscr{M}, u} \in \mathscr{X}_{S}$. If $k<l$, non-isolated stable configurations are $X_{A}, X_{B}$ and $X_{S}^{\mathcal{S}, v} \in \mathscr{X}_{S}$.

Proof. In this proof, the notation of proof of Theorem 1 is used. We assume that $k>l$ and prove that $X_{S}^{\mathscr{M}, u}, X_{A}$ and $X_{B}$ are non-isolated stable configurations. If a configuration $X$ of an $m \times n$ cell space is a non-isolated stable configuration, then at least there exists one basic cell space $B$ such that all cells of $B$ have the same state. Firstly, let this state be " $b$ " state. Then, all states of $S_{1}[X]$ and $S_{2}[X]$ must be " $b$ " state because $\left|S_{1}[X]\right|\left(\left|S_{2}[X]\right|\right)=l$ and $p>l$. States of any $p$ cells of $S_{3}[X]$ or $S_{4}[X]$ can be allocated " $a$ " states because $p \leqslant k$ and other cells' states are " $b$ ". Of course, we can allocate " $b$ " state to all cells of these cell subspaces. By considering the basic cell space $B_{1,3}=\left\{c_{r, s} ; i-1 \leqslant r \leqslant i+k-2, j-1 \leqslant s \leqslant j+l-2\right\}$, it is known that a state of cell $c_{i-1, j-1}$ depends on a state of $c_{i+k-1, j-1}$. If $c_{i+k-1, j-1}[X]=a(b)$, then $c_{i-1, j-1}[X]=a(b)$. By the similar considerations, we know that $c_{i+k, j-1}[X]=c_{i, j-1}[X]$, $c_{i-1, j+l}[X]=c_{i+k-1, j+l}[X]$ and $c_{i+k, j+l}[X]=c_{i, j+l}[X]$. The similar determination processes of states of cells can be continued step by step. Then, we get $X_{S}^{\mathcal{M}^{\mathcal{u}}} \in \mathscr{X}_{S}$ or $X_{B}$ as a whole. Secondly, we assume that all states in $B \in \mathscr{B}$ are " $a$ ". Then, by the similar arguments, we find that this configuration is $X_{A}$. In the case that $k<l$, we can prove this theorem by the similar method. Q.E.D.

Two examples for these non-isolated stable configurations under $[4,6]$-rule are shown in Fig. 1. Hereafter, in figures, a black cell in the cell space has " $a$ " state and a white cell has " $b$ " state. From these examples, it is clearly known that a direction of "a-state string" depends on the form of basic cell space because a basic cell space is $6 \times 5$ cell subspace in Fig. 1(a) and it is $5 \times 6$ cell subspace in Fig. 1(b).

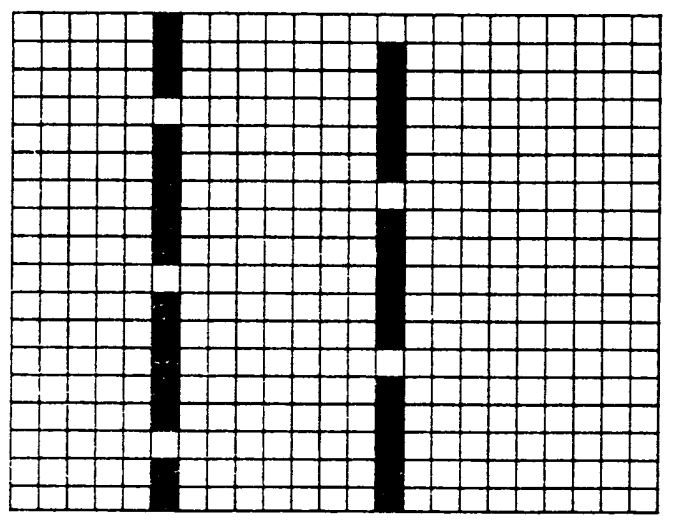

(8)

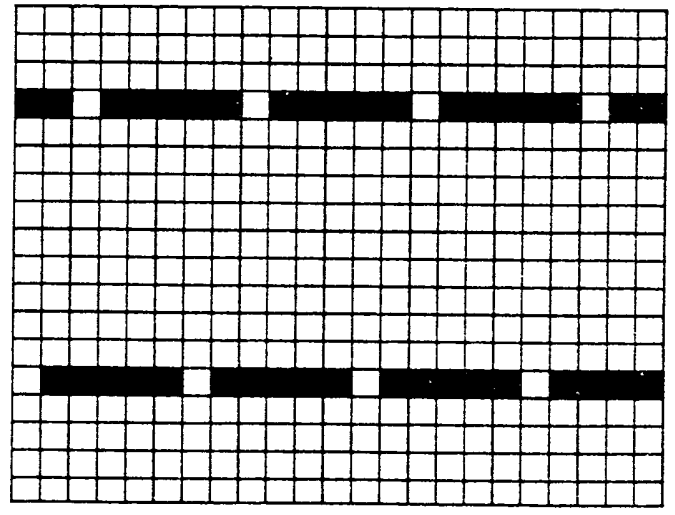

(b)

Fig. 1. Two examples of non-isolated stable configurations under $[4,6]$-rule. Hereafter, in all figures, a black cell has " $a$ " state and a white cell has " $b$ " state.

(a) A basic cell space is a $6 \times 5$ cell subspace.

(b) A basic cell space is a $5 \times 6$ cell subspace. 


\section{3. $1 \leqslant p \leqslant \min (k, l)$}

Two cases that $k=l=2$ and $k>2, l>2$ should be separately considered because if $k=l=2$, a stable configuration is a checked pattern and if $k>2, l>2$, then other patterns are non-isolated stable configurations. First of all, we consider the latter. In order to present stable configurations for $p=1$, we need a definition of a neighborhood of any cell. A cell subspace $U\left(c_{i j}\right)$ is said to be a neighborhood of $c_{i j}$ if and only if

$$
U\left(c_{i j}\right)=\bigcup_{B \in \mathscr{B}\left(c_{i j}\right)} B-\left\{c_{i j}\right\}
$$

where $\mathscr{B}\left(c_{i j}\right)$ is a set of all basic cell spaces that includes $c_{i j}$.

THEOREM 3. Under [0,2]-rule, stable configurations are $X_{A}, X_{B}$ and an a-state sporadic configuration which satisfies the condition that if $c_{i j}\left[X_{S}\right]=a$, then for any $c_{k l} \in U\left(c_{i j}\right), c_{k l}\left[X_{S}\right]=b$.

Proof. By using definition of [0,2]-rule, it is easily proved that $X_{A}, X_{B}$ and a-state sporadic configuration are stable configurations. The detail of proof is omitted.

Q.E.D.

An example for a-state sporadic configurations is shown in Fig. 2. When a rule is $[1,3]$-rule, a pattern of non-isolated stable configuration is significantly different from other one. In this pattern, it is possible that two "a-state strings" cross in " $b$-state sea".

THEOREM 4. If a rule is [1,3]-rule, then non-isolated stable configurations are $X_{A}$, $X_{B}, X_{S}^{\mathscr{M}, u}, X_{S}^{\mathcal{N}, v}$ and $X_{S}^{(\mathscr{M}, u)(\mathcal{N}, v)}$.

Proof. The method of proof is similar to the proofs of previous theorems. The detail of proof is omitted. Q.E.D.

An example of the above non-isolated stable configuration $X_{S}^{(\mathscr{M}, u)(\mathscr{N}, v)}$ is shown in Fig. 3. Under the condition $3 \leqslant p \leqslant \min (k, l)$, vertical or horizontal "a-state strings" exists in non-isolated stable configurations, but both kinds of these strings can not coexist in one non-isolated stable configuration, which is different from the case $p=2$. The vertical (horizontal) "a-state strings" exist whether $k<l$ or not. This is different from the case that $\min (k, l)<p \leqslant \max (k, l)$.

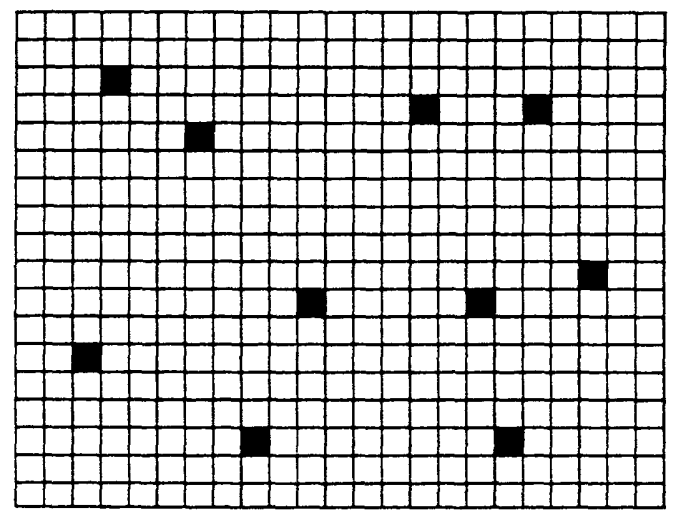

Fig. 2. An example of a-state sporadic stable configurations under $[0,2]$-rule in a cell space with $2 \times 3$ basic cell spaces. 


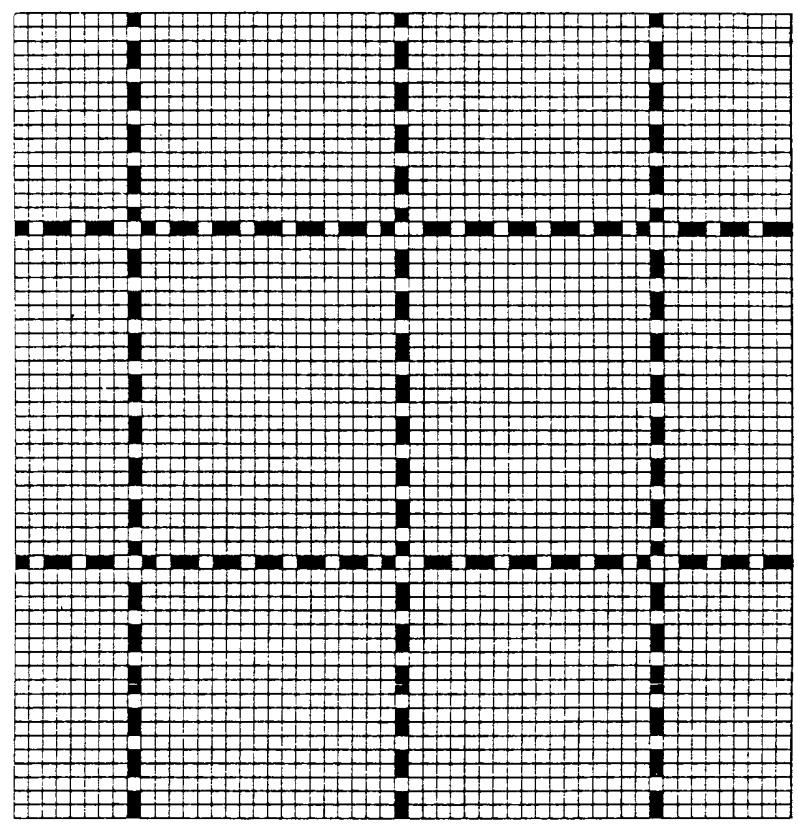

Fig. 3. An example of non-isolated stable configurations under [1,3]-rule in a cell space with $3 \times 3$ basic cell spaces.

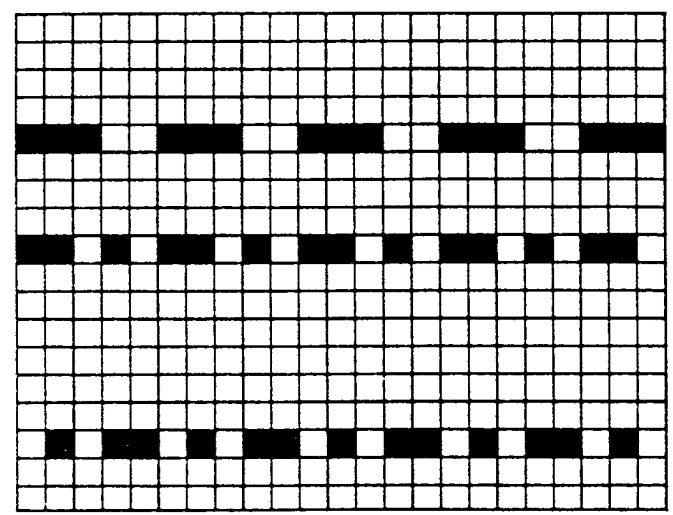

(a)

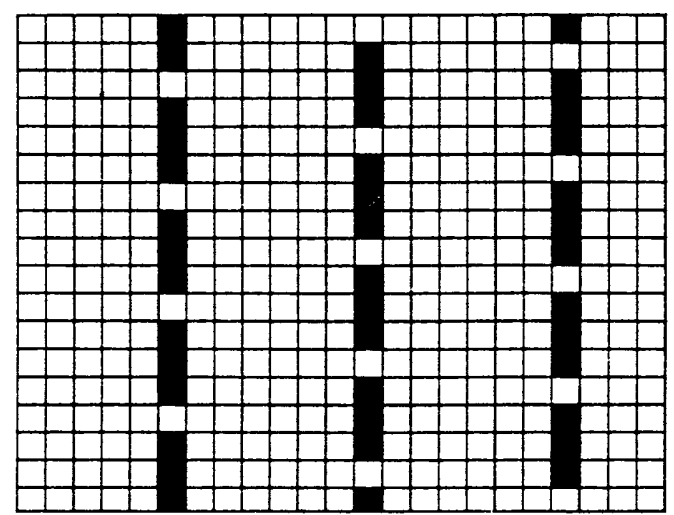

(b)

Fig. 4. Two examples of non-isolated stable configurations under $[2,4]$-rule in a cell space with $4 \times 5$ basic cell spaces.

THEOREM 5. If $3 \leqslant p \leqslant \min (k, l)$, then non-isolated stable configurations are $X_{A}, X_{B}$, $X_{S}^{\mathscr{M}, u}$ and $X_{S}^{\mathcal{L}, v}$.

Proof. By the similar method, we can prove the above theorem. The detail of proof is omitted. Q.E.D.

Two examples for non-isolated stable configurations under [2, 4]-rule are shown in Fig. 4. Lastly, we consider the case that $k=l=2$. If $p=1$, then stable configurations 
are $X_{A}, X_{B}$ and $a$-state sporadic stable configuration. If $p=2$, then stable configurations are well-known checked patterns which were obtained by T. Kitagawa and M. Yamaguchi $[4,5]$.

It is easily found that any stable configuration under $[p-1, p+1]$-rule is complementary to a stable configuration under $[k l-p-1, k l-p+1]$-rule. Here, a configuration $\bar{X}$ is said to be complementary to $X \in \mathscr{X}$ if and only if $c_{i j}[\bar{X}]=\overline{c_{i j}[X]}$ for any cell where $\bar{a}=b$ and $\bar{b}=a[5]$. By using this fact, we can get a pattern of a nonisolated stable configuration under other $[p-1, p+1]$-rule which are not presented in this section.

\section{Hierarchy of the Types of Local Transition Rules}

We introduce an order relation $\preceq$ into a set of all types of local transition rules. For two rules $\alpha$ and $\beta$, the order relation $\alpha \leq \beta$ exists if and only if any stable configuration under a rule $\alpha$ is also stable under a rule $\beta$. Furthermore, in the subset $\left\{\left[r_{1}, r_{2}\right]-\right.$ rule $\left.\mid r_{1}, r_{2} \neq 1, r_{2}-r_{1} \neq 1\right\}$ of the set of all rules, we can introduce the join $\alpha \oplus \beta$ which is defined by $\alpha \oplus \beta=\sup \{\alpha, \beta\}$. This join is used in the next section.

THEOREM 6. The following order relations exist.

$$
\begin{gathered}
{[\perp, 0] \preceq[q-1, q]} \\
{[k l, \perp] \preceq[q-1, q]} \\
{[q-1, q] \preceq[p-1, p+d]}
\end{gathered}
$$

where $1 \leqslant q \leqslant k l-1,1 \leqslant p \leqslant k l-1$ and $1 \leqslant d \leqslant k l-p$.

Proof. Under $[\perp, 0]([k l, \perp])$-rule, $X_{A}\left(X_{B}\right)$ is only one stable configuration and under $[q-1, q]$-rule, all stable configurations are $X_{A}$ and $X_{B}$. Furthermore, $X_{A}$ and $X_{B}$ are stable under $[p-1, p+d]$-rule. From these facts, we obtain Theorem 6 .

Q.E.D.

THEOREM 7. An order relation $[p-1, p+d] \preceq\left[p^{\prime}-1, p^{\prime}+d^{\prime}\right]$ exists if and only if $d<d^{\prime}, p^{\prime}<p, p+d \leqslant p^{\prime}+d^{\prime}$.

ProOF. From transition tables of $[p-1, p+d]$-rules, we can easily prove this theorem. The detail of proof is omitted. Q.E.D.

\section{Cluster Property of Stable Configurations}

From Theorem 7 , we obtain that $[q-1, q+1] \leq[p-1, p+d](p \leqslant q \leqslant d+p-1)$. Thus, any stable configuration $X_{S}$ under $[p-1, p+d]$-rule $(d \geqslant 2)$ consists of the union of local stable configurations $B\left[X_{S}\right](B \in \mathscr{B})$, which are stable under $[q-1, q+1]$-rule $(p \leqslant q \leqslant d+p-1)$. Simply, we can represent this fact as

$$
[p-1, p+d]=[p-1, p+1] \oplus[p, p+2] \oplus[p+1, p+3] \oplus \cdots \oplus[p+d-2, p+d] .
$$

In an $m \times n$ cell space with $[p-1, p+d]$-rule, a cell subspace whose configuration can not be changed by $[i-1, i+1]$-rule $(p \leqslant i \leqslant d+p-1)$ is called $[i-1, i+1]$ domain which is denoted by $D_{i}$. Our interest is possible distribution of these domains. 
$[i-1, i+1]$ and $[j-1, j+1]$ domains are said to be able to overlap with an overlap index $\kappa_{i j}$ if and only if there exist basic cell spaces $B$ and $B^{\prime}$ such that $B$ and $B^{\prime}$ belong to $[i-1, i+1]$ and $[j-1, j+1]$ domains respectively, $\left|B \cap B^{\prime}\right|=\kappa_{i j} \neq 0$ and configurations of $B$ and $B^{\prime}$ are not uniform.

THEOREM 8. In an $m \times n$ cell space with $k \times l$ basic cell space, $[p-1, p+1]$ domain and $[k l-r-1, k l-r+1](p<k l-r)$ domain can not overlap with an overlap index $\kappa_{p, k l-r}=w$ if and only if $w>p$ and $r<w-p . \quad[p-1, p+1]$ domain and $[k l-r-1, k l-r+1]$ domain can overlap with an overlap index $w$ if and only if $w \leqslant p$.

Proof. We assume that $w>p$ and $B \cap B^{\prime} \neq \phi$ where $B \subset D_{p}$ and $B^{\prime} \subset D_{k l-r}$. We can allocate " $a$ " state to $p-x$ cells and " $b$ " state to other $w-p+x$ cells in $B \cap B$ '

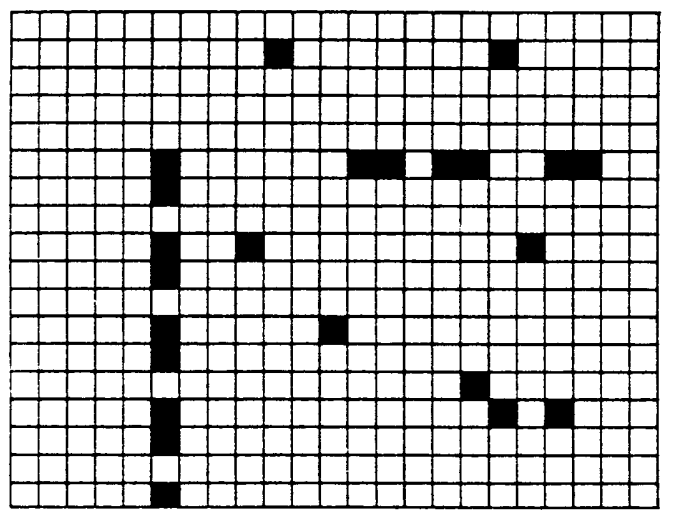

(a)

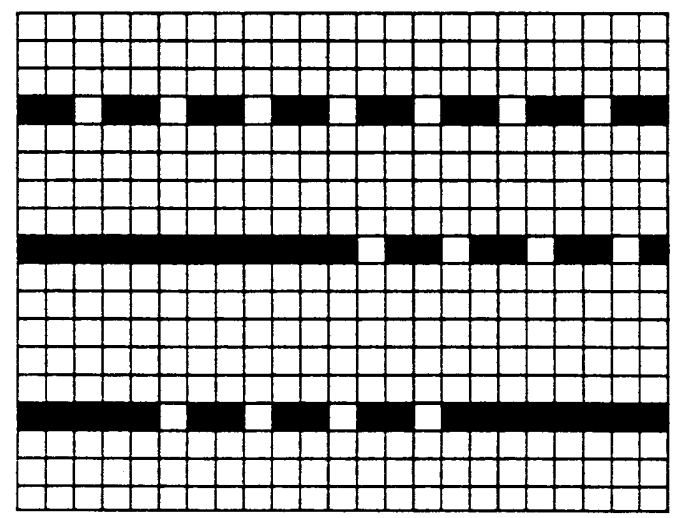

(b)

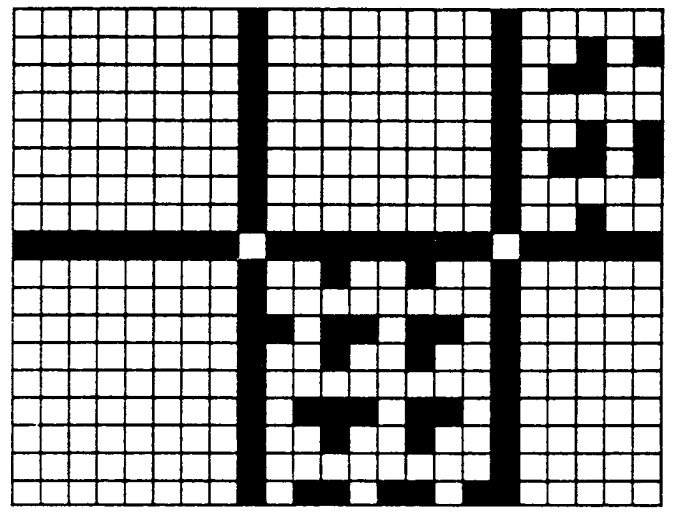

(c)

Fig. 5. Three examples of cluster property of stable configurations under $[p-1, p+2]$-rule $(p=1,2,3)$.

(a) A stable configuration under [0,3]-rule.

(b) A stable configuration under [1,4]-rule.

(c) A stable configuration under [2,5]-rule. 
where $0 \leqslant x<p$. If " $a$ " state is allocated to $x$ cells and " $b$ " state is allocated to other cells in $B-B \cap B^{\prime}$, a configuration of $B$ is stable. However, if $r<w-p+x$, a configuration of $B^{\prime}$ is not stable. Thus, if $r<w-p$, then two domains $D_{p}$ and $D_{k l-r}$ can not overlap with an overlap index $w$. Secondly, we assume that $w \leqslant p$. The " $a$ " state is allocated to $w-x$ cells and the " $b$ " state is allocated to other $x$ cells in $B \cap B^{\prime}$ where $0 \leqslant x \leqslant w$. Then, there exist two stable configurations of $B$ and $B^{\prime}$ if and only if $k l \geqslant p+x$ and $k l-w \geqslant r-x$. There exists the number $x$ which satisfies these conditions. From these calculations, Theorem 8 is proved. Q.E.D.

Examples of cluster property of stable configurations are shown in Fig. 5. In Fig. 5 , under three rule $[p-1, p+2](p=1,2,3)$ and $3 \times 3$ basic cell space, stable configurations are shown.

\section{Singular Stable Subconfiguration}

In an $m \times n$ cell space, by a change of state of only one specific cell, a stable configuration may not be changed into another stable configuration. For investigating these facts, we define the singular stable subconfiguration.

Definition 1. In an $m \times n$ cell space $C$, for $X_{S} \in \mathscr{X}_{S}$ and $V \subseteq C, V\left[X_{S}\right]$ is said to be the singular stable subconfiguration iff $V\left[X_{S}\right]$ satisfies the following conditions:

1. If for any cell $c_{x y} \in V, X^{\prime}=\left\{c_{i j}\left[X^{\prime}\right] \mid c_{i j}\left[X^{\prime}\right]=c_{i j}\left[X_{S}\right](i \neq x, j \neq y), c_{x y}\left[X^{\prime}\right]=\right.$ $\left.\overline{c_{x y}\left[X_{S}\right]}\right\}$, then

$$
\operatorname{Prob}\left(X^{\prime} \rightarrow Y\right) \neq 0
$$

where $Y \neq X_{S}, X^{\prime}$ and $Y \in \mathscr{X}$.

2. If for any cell $c_{z w} \in C-V, X^{\prime \prime}=\left\{c_{i j}\left[X^{\prime \prime}\right] \mid c_{i j}\left[X^{\prime \prime}\right]=c_{i j}\left[X_{S}\right] \quad(i \neq z, j \neq w)\right.$, $\left.c_{z w}\left[X^{\prime \prime}\right]=\overline{c_{z w}\left[X_{S}\right]}\right\}$, then

$$
\operatorname{Prob}\left(X^{\prime \prime} \rightarrow X_{S}\right)=1
$$

We can easily obtain the singular stable subconfiguration of each stable configuration under any type of local transition rule, but we omit the long list of singular stable subconfigurations. However, here, we will discuss biomathematical implication of the above singular stable subconfiguration in short. Any small change of the singular stable subconfiguration induces a large alternation of stable configuration because the interaction between cells maintains this singular stable subconfiguration. In biological phenomena, similarly, a small change of gene or DNA of eucaryote induces an alteration of phenotypic expression or function of organism. We think that a gene is the subsystem of whole system of organism, which is similar to the singular stable subconfiguration. We insist that a gene can be interpreted as "singular subsystem" rather than organism's blueprint where "singular subsystem" is an extension of the singular stable subconfiguration. An example of the singular stable subconfiguration is shown in Fig. 6 where a set of dotted and black cells' states is a singular stable subconfiguration. The transition from the configuration which is obtained from a small change of the singular stable subconfiguration is shown in Fig. 7. 


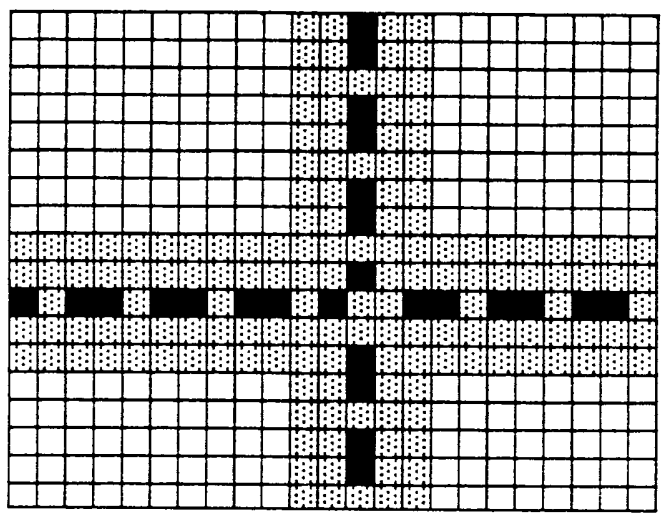

Fig. 6. An example of singular stable subconfigurations under [1,3]-rule in a cell space with $3 \times 3$ basic cell spaces. A set of dotted and black cells' states is a singular stable subconfiguration where a black cell's state is " $a$ " and other cells' states are " $b$ ".
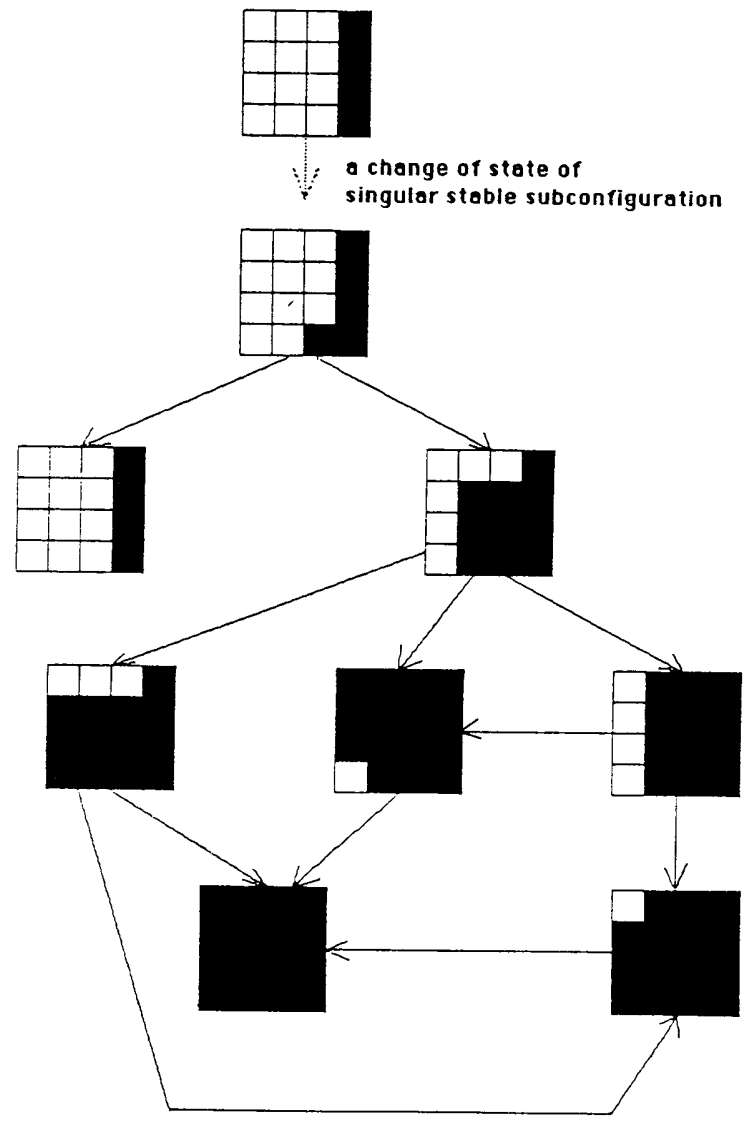

Fig. 7. A lineage of transition from a configuration with damaged singular stable subconfiguration. The original configuration is stable under $[2,4]$-rule in a $4 \times 4$ cell space with $3 \times 3$ basic cell spaces. 


\section{Patterns of Isolated Stable Configurations}

Finally, we investigate an isolated stable configuration which does not have any direct ancestor. It is easily found that under $[\perp, 0]$ and $[k l, \perp]$-rule, there does not exist an isolated stable configuration and under $[0,1]([k l-2, k l-1])$-rule, an isolated stable configuration is only $X_{B}\left(X_{A}\right)$. However, under $[p-1, p+d]$-rule, patterns of isolated stable configurations are more complex than patterns of non-isolated stable configurations. Here, we show some examples of these isolated stable configurations under $[1,3]$-rule in Fig. 8.

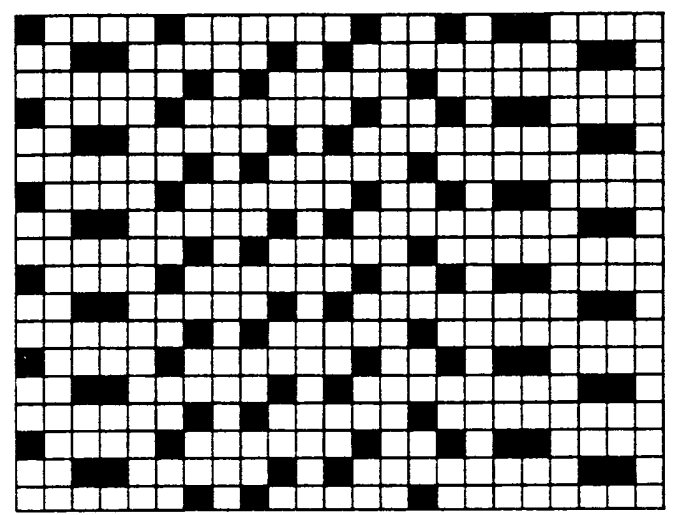

(a)

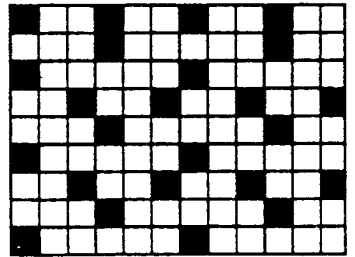

(b)

Fig. 8. Two examples of isolated stable configurations under [1,3]-rule in a cell space with $3 \times 3$ basic cell spaces.

(a) In the row direction, this isolated stable configuration is periodic.

(b) In any direction, this isolated stable configuration is not periodic.

\section{Summary and Discussion}

In this paper, we have proposed the threshold type transition rule. Then, we have obtained all types of local transition rule, which is called a rule for short. We have found all patterns of non-isolated stable configurations in the cell space with $k \times l$ basic cell space under these rules. We have defined the singular stable subconfiguration and discussed its biomathematical meaning.

In the cell space, the interaction between cells or the transition rule is not symmetric in respect to each cell. Thus, our cell space is different from usual cellular automata, for example, as famous von Neumann's cellular automata and Wolfram's two-dimensional cellular automata [10].

Each rule or type of local transition rule has robustness against small perturbations of parameters in a rule $L T$. This means that even if parameters of the transition rule $L T$ suffer small perturbations, the type of local transition rule remains unchanged. This statement is equivalent to another statement that broad spectra of parameters of 
the transition rule give us the same type of local transition rule. We think that this concept of robustness of a rule is also important when we understand biological phenomena. By thermodynamic fluctuation and external disturbance, organized structures of life are usually at a crisis. We assume that the interaction between elements of organized structure depends on some parameters and these parameters are perturbed by foregoing fluctuations. However, in order to maintain organized structures, the result of interactions must not change even if the parameter of interaction is disturbed by these fluctuations. Thus, we believe that robustness of rule is one of important concepts in biomathematics.

\section{Acknowledgments}

The author would like to thank Dr. T. Kitagawa, the former president of International Institute for Advanced Study of Social Information Science, Fujitsu Limited, for suggesting the definition of the local transition rule and valuable discussions. $\mathrm{He}$ would also like to express Dr. M. Toda for critical reading of the manuscript. He is also deeply indebted to Dr. K. Matsuo for valuable discussions. He would also like to thank Dr. K. Tajima for critical reading of the manuscript. He is much indebted to the referee for helpful comments about Theorem 1, Theorem 5 and the notation of rules.

\section{References}

[1] von Neumann, J.: In the theory of self-reproduced automata, (A. W. Burks, ed.) Pt. II. Univ. of Illinois Press, Urbana, Illinois, (1966).

[2] Wolfram, S. ed.: Theory and application of cellular automata, World Scientific. Singapore, (1986).

[3] Kitagawa, T.: A contribution to the methodology of biomathematics, Math. Biosci., 12 (1971), 329-345.

[4] Kitagawa, T.: Cell space approaches in biomathematics, Math. Biosci., 19 (1974), 27-71.

[5] Kitagawa, T. and Yamaguchi, M.: Local majority transormations in cell space, Bull. Math. Stat., 14 (1971), 61-82.

[6] Yamaguchi, M.: Stable configurations under local majority transformation in triangular cell space, Bull. Math. Stat., 14 (1971), 93-106.

[7] Kitagawa, T.: Prolegomena to cell space approaches, Mem. Fac. Sci. Kyushu Univ., A26 (1971).

[8] Kitagawa, T.: Generative and genealogical classifications of all the configurations in an $m \times n$ cell space under applications of local majority transformation, Bull. Math. Stat., 15 (1-2) (1972), 85-112.

[9] SASAGAWA, F.: The dynamics on the cell spaces with $p$ states under the local transformations satisfying the principle of local majority, Bulletin of Informatics and Cybernetics, 23 (3-4) (1989).

[10] Packard, N. H. and Wolfram, S.: Two-dimensional cellular automata, Journal of Statistical Physics, 38 (5-6) (1985), 901-946.

Received April 21, 1989

Revised July 3, 1989

Communicated by T. Kitagawa 